\title{
THE SPIN-WAVE STIFFNESS \\ WITHIN DENSITY FUNCTIONAL THEORY AND THE GINZBURG-LANDAU FUNCTIONAL
}

\author{
W. SCHMIDT* \\ Institute of Molecular Physics, Polish Academy of Sciences \\ Smoluchowskiego 17, 60-179 Poznań, Poland
}

\begin{abstract}
The stiffness constant of the stationary spin excitation is calculated employing density functional theory and the phenomenological GinzburgLandau energy functional in a site form. The exchange parameter of the magnetic functional is estimated within the framework of the nearest neighbours intermolecular interaction and the energy gain of non-magnetic state per atom. The stiffness constant of $\mathrm{Fe}, \mathrm{Co}$, and Ni metals is found based on the energies of spin polarization calculated numerically in the local spin density approximation by Moruzzi et al. as well as by Kübler. The calculated stiffness constants are compared with other theoretical calculations and experimental data.
\end{abstract}

PACS numbers: 71.15.Mb, 75.30.Et

\section{Introduction}

In the past years there has been a development of theory of non-collinear magnetic states (for a review, see [1]) and calculations of magnon spectra [2-4] within the framework of classical static density functional theory (DFT) of Hohenberg and Kohn (HK) [5] and Kohn and Sham (KS) [6] and the Barth and Hedin extension [7] to the spin polarized case. A tool for studying dynamic interactions, as well as for the computation of excitation energies is the time dependent DFT of Runge and Gross [8]. To calculate the KS potentials of a stationary excitation, it is purposeful to employ the exchange-correlation (xc) energy functional $E_{\mathrm{xc}}\left[\rho_{\uparrow}, \rho_{l}\right]$ of the ordinary density functional theory [9]. The KS Hamiltonian has the form $[1,7]$ :

$$
H_{\mathrm{KS}}=\left(-\frac{\hbar^{2}}{2 m} \nabla^{2}+V_{\mathrm{eff}}\right) \hat{\mathrm{I}}-\mu_{\mathrm{B}} B_{\mathrm{xc}} U^{+}(\vartheta, \varphi) \widehat{\sigma}_{z} U(\vartheta, \varphi) .
$$

\footnotetext{
*corresponding author; e-mail: schmidt@ifmpan.poznan.pl
} 
Here, $\hat{1}$ and $\widehat{\sigma}_{z}$ are, respectively, the unit and Pauli matrices. The effective potential and the $\mathrm{xc}$ field are functionals of the up and down densities: $V_{\text {eff }}=V_{\text {eff }}\left[\rho_{\uparrow}, \rho_{\downarrow}\right](r)$, $B_{\mathrm{xc}}=B_{\mathrm{xc}}\left[\rho_{\uparrow}, \rho_{\downarrow}\right](r)$. A time dependence of $H_{\mathrm{Ks}}$ is through the spin $\frac{1}{2}$ rotation matrix $U(\vartheta, \varphi)$. Polar angles $\vartheta$ and $\varphi$ of the direction of spin polarization $m(r, t)$ specify the stationary spin excitation. The arrangement $\{\vartheta, \varphi\}$ gains a status of spin-wave parameter. The vector $\{\vartheta, \varphi\}$ can be taken uniform within the atomic cell, and for a static state is constant and describes a non-collinear structure, or a Bloch wall. For a flat spin-wave, the spin arrangement parameter $\{\vartheta, \varphi\}$ is confined to the form: $\vartheta=$ const and $\varphi=\omega_{q} t-r q_{l}$. Here, $\omega_{q}$ and $q$ are, respectively, the frequency of spin-wave and the magnon wave vector. In principle, the magnetic stationary excitation could be determined within a self-consistent calculation of the time dependent Kohn-Sham and the torque Landau-Lifschitz equations. This is cumbersome and the Ginzburg-Landau magnetic functional is employed calculating the functional parameters in a static limit.

\section{The exchange parameter}

In the spirit of DFT, the total energy expression of a stationary magnetic state has to be a functional of the electronic density, $\rho=\rho(\boldsymbol{r})$, and the spin polarization $m=m(r, t)$. The Ginzburg-Landau (GL) magnetic functional is the simplest example. In the classic GL approach $[10,11]$, the total energy expression is a functional of the magnetization $M=-g \mu_{\mathrm{B}} S_{l} / V_{l}$ and a gradient contribution of $\boldsymbol{M}$. Here, $g$ is the spectroscopic splitting factor, $\boldsymbol{S}_{l}$ and $V_{l}$ are, respectively, the site spin and the site volume assigned to the $l$-th cell. In the frame of GL functional, the energy gain of a magnetic stationary excitation is approximated by the site form

$$
\begin{aligned}
E_{\mathrm{ex}} & =E[\rho, m]-E\left[\rho_{0}, m_{0}\right] \approx a \sum_{l}\left(S_{l}^{2}-S_{0}^{2}\right)+b \sum_{l}\left(S_{l}^{4}-S_{0}^{4}\right)+\ldots \\
& -g \mu_{\mathrm{B}} B_{0} \sum_{l}\left(S_{l}^{(z)}-S_{0}\right)-\sum_{l} \sum_{\delta} J(\delta)\left(\boldsymbol{S}_{l} \cdot S_{l+\delta}-S_{l}^{2}\right) .
\end{aligned}
$$

For a stationary state, the total energy $E[\rho, m]=E\left[\rho_{\uparrow}, \rho_{\downarrow},\{\vartheta, \varphi\}\right]$ depends on the parameter $\{\vartheta, \varphi\}$ both explicitly and implicitly via the up and down electronic densities $\rho_{\uparrow}=\rho_{\uparrow}[\{\vartheta, \varphi\}](r)$ and $\rho_{\downarrow}=\rho_{\downarrow}[\{\vartheta, \varphi\}](r)$. The total energy is minimized with respect to the electronic densities $\rho_{\uparrow}$ and $\rho_{\downarrow}$. In the ground state, the densities and the total energy are, respectively, $\rho_{0}=\rho_{0}(r), m_{0}=m_{0}(r)$ and $E\left[\rho_{0}, m_{0}\right]$. The expansion coefficients: $a, b$ and the exchange parameter $J(B)$ can be provided by the DFT calculations. Small correction of the orbital momentum is neglected and the factor $g=2$. The $z$-direction of the global coordinate system is specified by the external magnetic field, $\boldsymbol{B}_{0}=\left(0,0,-B_{0}\right)$, which usually is assumed to be zero. In the ground state, the site spins are $S_{0}$.

Our numerical discussion of a stationary spin excitation, within DFT and the phenomenological energy functional (2), will be confined to the intermolecular interaction between nearest neighbour pairs and the approximation: $S_{l}^{2} \approx S_{0}^{2}$. This approximation sets up a simple relation between the energy gain of (2) of 


\section{TABLE}

$D_{\text {cal }}, D_{\text {expt }}$, and $D_{\text {ref }}$ are respectively, the present calculated, reference measured and reference calculated values of the magnon stiffness constant at low temperatures, in mRy bohr ${ }^{2}$. Here, $E_{\text {exo }}$ (in $\mathrm{mRy}$ ) and $S_{0}$ are, respectively, the energy gain of non-magnetic state per atom and the site spin of Ref. [13] and [2] which are applied in the present calculations.

\begin{tabular}{c|c|c|c|r|r}
\hline \hline Metal & $D_{\text {cal }}$ & $E_{\text {exo }}$ & \multicolumn{1}{c|}{$2 S_{0}$} & \multicolumn{1}{|c|}{$D_{\text {ref }}$} & $D_{\text {expt }}$ \\
\hline bcc Fe & $136^{a}$ & $21^{a}$ & $2.15^{a}$ & $65[4]$ & $87[14]$ \\
& $79^{b}$ & $12.5^{b}$ & $2.2^{b}$ & $93[2]$ & $82[15]$ \\
fcc Co & $73^{a}$ & $8^{a}$ & $1.56^{a}$ & $132[4]$ & $122[16]$ \\
& $131^{b}$ & $14.8^{b}$ & $1.61^{b}$ & $140[2]$ & $134[15]$ \\
fcc Ni & $146^{a}$ & $6^{a}$ & $0.59^{a}$ & $194[4]$ & $147[17]$ \\
& $144^{b}$ & $6.1^{b}$ & $0.61^{b}$ & $207[2]$ & $144[15]$ \\
\hline
\end{tabular}

${ }^{a}$ Ref. [13]; ${ }^{b}$ Ref. [2].

non-magnetic state per atom and the exchange parameter, cf. [12]. For literature values $[13,2]$ of the energy gain of non-magnetic state per atom, the magnon stiffness constant values have been found and are shown in Table. The present results are in a fair agreement with experimental data. The calculated stiffness constant within [13] seems to be not so close to experiment as the calculation based on [2], since the approximation: $S_{l}^{2}=S_{0}^{2}$, is postulated for the paramagnetic state. To calculate dynamical properties of the ferromagnetic $3 d$ metals we must take into account an effect of spin-fluctuations on the site spin momentum [4]. The energy gain of non-magnetic state per atom of [2] is approximated by a superposition of spiral states with the constrained site spin, $S_{l}^{2}=S_{0}^{2}$ and $\vartheta=\pi / 2$. This gives the astonishing agreement with experimental low temperature data of the stiffness constant in Table. For the itinerant model with the Hubbard Hamiltonian, the calculated stiffness constant [16] of fcc Co is $212 \mathrm{mRy} \mathrm{bohr}{ }^{2}$, and for bcc Fe and fcc Ni [18] are, respectively, 147 and $174 \mathrm{mRy} \mathrm{bohr}^{2}$. The agreement of the latter seems to be not so close to experiment as the present one, since the itinerant model neglects the effect of spin-waves on the intermolecular distance (invar effect).

\section{Acknowledgments}

I would like to acknowledge some discussion with J. Morkowski and A.R. Ferchmin. This work was supported by the grant of the Committee for Scientific Research No. 2P03B 11814. 


\section{References}

[1] L.M. Sandratskii, Adv. Phys. 47, 91 (1998).

[2] J. Kübler, Acta Phys. Pol. A 97, 165 (2000).

[3] N.M. Rosengaard, B. Johansson, Phys. Rev. B 55, 14975 (1997).

[4] S.V. Halilov, H. Eschrig, A.Y. Perlov, P.M. Oppeneer, Phys. Rev. B 58, 293 (1998).

[5] P. Hohenberg, W. Kohn, Phys. Rev. 136, B864 (1964).

[6] W. Kohn, L.J. Sham, Phys. Rev. 140, A1133 (1965).

[7] U. von Barth, L. Hedin, J. Phys. C, Solid State Phys. 5, 1629 (1972).

[8] E. Runge, E.K.U. Gross, Phys. Rev. Lett. 52, 997 (1984).

[9] E.K.U. Gross, W. Kohn, Adv. Quant. Chem. 21, 255 (1990).

[10] G.G. Lonzarich, L. Taillefer, J. Phys C, Solid State Phys. 18, 4339 (1985).

[11] D. Wagner, J. Phys., Condens. Matter 1, 4635 (1989).

[12] W. Schmidt, Acta Phys. Pol. A 97, 967 (2000).

[13] V.L. Moruzzi, J.F. Janak, A.R. Williams, Calculated Electronic Properties of Metals, Pergamon, New York 1978.

[14] C.S. Wang, R.E. Prange, V. Korenman, Phys. Rev. B 25, 5766 (1982).

[15] K. Tajima, Y. Ishikawa, P.J. Webster, M.W. Stringfellow, D. Tocchetti, K.R.A. Zeabeck, J. Phys. Soc. Jpn. 43, 483 (1977).

[16] X. Liu, M.M. Steiner, R. Sooryakumar, G.A. Prinz, R.F.C. Farrow, G. Harp, Phys. Rev. B 53, 12166 (1996).

[17] J.W. Lynn, H.A. Mook, Phys. Rev. B 23, 198 (1981).

[18] C.S. Wang, R.E. Prange, V. Korenman, Phys. Rev. B 25, 5766 (1982). 